\title{
Vehikel, Charakter-Pendant und Mittel zur Raumerkundung
}

\author{
Das Auto als multifunktionales Strukturelement im Tatort
}

ROLF PARR

\section{BitTE EINSTEIGEN}

Wenn es einen Gegenstand gibt, der in nahezu jeder Tatort-Folge (und im Weiteren in nahezu jeder populären Fernsehserie) präsent ist, dann ist es das Auto. Das könnte man zunächst einmal rein statistisch sehen und auf Heike Klippel und Hartmut Winkler zurückgreifen, die festgestellt haben, dass »[z]wei Prozent aller Fernsehbilder an- oder abfahrende Autos« zeigen, »wobei auf ein abfahrendes Auto mit signifikanter Häufigkeit eine Überblendung und dann die Ankunft an einem anderen Ort folgen wird « (1994: 129). Das mag stimmen, und die Prozentzahl mag seit 1994 mit der allgemeinen Tendenz zu mehr und zu schnellerer Bewegung in Fernsehfilmen sogar noch etwas angewachsen sein. Ein solcher Befund sagt jedoch noch nichts über das breite Spektrum an Funktionen aus, die Autos in einer Krimireihe wie Tatort übernehmen können, und auch nichts über ihre Einbettung in gesellschaftsgeschichtliche Kontexte und schon gar nichts darüber, was Autos möglicherweise mit dem seriellen Charakter des Tatort zu tun haben. ${ }^{1}$

Autos sind nämlich nicht nur weitverbreitete Alltagsgegenstände mit instrumenteller Funktion. Vielmehr stellen sie als gesellschaftlich-kulturelle Dinge stets auch hochgradig mit >Bedeutung < aufgeladene Zeichenkomplexe mit vielfach alltagsweltlichem Bezug dar, die besonders gut für Analogierelationen jeglicher Art her-

1 Da die Verwendung von $>$ Reihe $<$ und $>$ Serie $<$ in Bezug auf den Tatort in der Forschung alles andere als einheitlich ist, soll hier unter der $>$ Reihe Tatort $<$ der Gesamtzusammenhang aller lokalen/regionalen Tatort-Serien verstanden werden; von einer einzelnen Sendung wird als >Folge $<$ gesprochen. 
angezogen werden können, v.a. in Form von Metaphern und kollektiv versteh- und benutzbaren Symbolen (vgl. Link/Reinecke 1987; Parr 1998, 1999, 2012). So kann es im Alltagsgespräch etwa heißen, man habe im Urlaub endlich einmal innerlich abgebremst<, in den Medien, dass die Bundeskanzlerin >in der Euro-Frage Gas gegeben habe< oder dass für diesen oder jenen Minister das >Ende seiner Dienstfahrt< drohe, und in einem Drama wie Horváths Kasimir und Karoline, dass »ein Weib « wie »ein Auto« funktioniere (Horváth 1994: 132).

Zudem können Autos gerade in Fernsehkrimis und speziell solchen des Typs Tatort vielfältige dramaturgische Funktionen übernehmen, die von der Figurencharakterisierung und der Entwicklung der Handlung über die regionale Verankerung und die Erkundung von Räumen bis hin zur Herstellung imaginärer Einheiten des Ortes reichen, so dass die Vehikel insgesamt zu multifunktionalen Strukturelementen werden, auf die kaum ein Regisseur verzichten mag. Was für den Drehbuchschreiber im Ermittler-Büro das Telefon ist (vgl. Bierbaum 2001), nämlich eine Art dramaturgischer Joker, über den beliebige Inhalte und Figuren sowie Vorkommnisse in das Geschehen eingebracht werden können, das ist außerhalb des Büros das Auto.

Beides zusammen, die Übersemantisierung von Autos und Autofahrten und ihre dramaturgische Nutzung, erklärt dann auch, warum es kaum eine Folge ohne Autofahrt, kaum einen Täter ohne Fluchtwagen, kaum einen Kriminalkommissar ohne zu ihm passendes Auto (mit Ausnahme des fast durchweg Fahrrad fahrenden Palu in Saarbrücken, den dann der Münsteraner Thiel beerbt hat) und kaum einen Polizeibeamten gibt, der nicht hinter einem Auto in Deckung geht. >Harry, hol schon mal den Wagen! ${ }^{2}$ ist der wahrscheinlich bekannteste Satz zur Fernsehserie Derrick (1974-1998), wenn auch nicht aus ihr, denn er ist so nie gesagt und schon deshalb gar nicht so oft wiederholt worden, wie man immer annimmt. Allerdings würde er den Stellenwert, den Zuschauer, Drehbuchschreiber und Regisseure dem Auto im Serienkrimi beimessen, sehr gut illustrieren, denn sonst würde das >Wagenholen nicht in anderen Krimiserien immer wieder einmal adaptiert. So spielt in Borowski und der coole Hund (2011) die Assistentin Sarah Brandt darauf an, wenn sie - in einer ihr entgleitenden Verhörsituation gebeten, schon einmal hinauszugehen - die Rolle des Derrick-Assistenten ironisch auf sich selbst anwendet und sagt: »Ich fahr' dann schon mal den Wagen vor.«(00:23:38-00:23:50) Eine ganz ähnliche Stelle findet sich auch 1998 in Bildersturm. Hier bittet Max Ballauf seinen Kollegen Freddy Schenk, schon einmal vorzugehen. Der erwidert ironisch: »Ach, Harry, hol schon mal den Wagen, oder was? «(00:21:20) Fortgeführt wird die damit aufgemachte Analogie zu Derrick dann noch einmal eine Minute später, als Schenk Ballaufs Hinweis auf sein zu schnelles Fahren mit »Entschuldigung, Stephan!«

2 Vgl. dazu Legal/Saure 1998. 
(00:22:24) kontert. ${ }^{3}$ Solche Bezugnahmen im Tatort sind serienübergreifende Formen der Selbstthematisierung des seriellen Charakters.

Wie das Spektrum der Funktionen, die Autos im Tatort einnehmen, genauer aussieht, soll im Folgenden aufgezeigt werden, wobei stets begleitend $\mathrm{zu}$ fragen sein wird, inwiefern das Strukturelement >Auto< über die filminternen Funktionen und seine gesellschaftsgeschichtliche Einbettung hinaus auch etwas mit der Serialität des Tatort zu tun hat.

\section{FAHRT AUFNEHMEN: KOMMISSARE UND IHRE AUTOS}

Durch Analogien, die - wie in den eingangs angeführten Beispielen - Autos und Menschen bzw. menschliches Verhalten in Relation zueinander setzen, werden nicht nur Menschen durch die Brille des Vehikels angeschaut, sondern in Umkehr der Blickrichtung stets auch die Autos anthropomorphisiert. Von daher verwundert es nicht, dass beide Bereiche immer wieder aufeinander abgebildet werden: dies nicht nur im Fernsehkrimi, sondern auch in allen anderen medialen und literalen Genres, beginnend bei Bilderbüchern wie Hoppla Hugo, der Geschichte eines kleinen Taxis, mittels dessen die lafontaineschen Fabeln für Kinder >automobil< nacherzählt werden (Guillaume/Roca 2003). Die Abbildung von Autos auf Menschen und umgekehrt macht es möglich, dass die Vehikel der Tatort-Kommissarinnen und -Kommissare bevorzugt zur Charakterisierung ihrer Fahrer dienen. ${ }^{4}$ Das leuchtet unmittelbar ein, wenn man für die Kölner Tatort-Serie an Kommissar Freddy Schenk und seine Vorliebe für große amerikanische Youngtimer denkt, die für seinen Partner Max Ballauf völlig undenkbar wären. Mit dem üppigen Körper Schenks korrespondieren die mit nicht minder üppiger Körperlichkeit daherkommenden amerikanischen Wagen, und zwar stets solche, die im kriminellen Milieu konfisziert wurden und jetzt auf der anderen Seite des Gesetzes den Kommissar zieren. Mal ist das »eine 64er Corvette«, mal ein »1966 Plymouth Valiant Signet Convertible ${ }^{5}{ }^{5}$

3 Für den Hinweis auf diese Stelle danke ich François Werner von www.tatort-fundus.de.

4 Vgl. Frank Rauscher. » Tatort<-Kommissare und ihre Autos. Nur manche mögen's heiß« (http://www.tatort-fundus.de/web/lexikon/autos-der-ermittler.html). Konsequent zu Ende gedacht hat das Prinzip der Anthropomorphisierung von Autos die US-amerikanische Serie Knight Rider (1982-1986), in der besondere Autos sprechen und gelegentlich sogar quasi-emotional reagieren können.

5 [Anon.] »Dienstwagen-Affären - Die Karossen der >Tatort<-Kommissare.« klamm.de, 5. Dezember 2011 (http://www.klamm.de/news/dienstwagen-affaeren-die-karossen-dertatort-kommissare-30N855248765.html). 
Gelegentlich gibt es sogar regelrechte Testsituationen darauf, wie stabil die Kopplung von Auto und Kommissar für die Fernsehzuschauer ist. In dem in Köln und Leipzig spielenden Tatort-Doppel Kinderland und Ihr Kinderlein kommet von 2012 fährt Schenk zusammen mit seiner Leipziger Kollegin Eva Saalfeld zunächst in seinem amerikanischen Straßenkreuzer (Ihr Kinderlein kommet, 01:03:4801:04:10), was diese sichtlich goutiert, nicht aber ihr Leipziger Partner und ExEhemann Keppler. Später überlässt Schenk ihr dann sogar den Wagen, was im Falle seiner Kölner Kollegen undenkbar gewesen wäre, denn denen gegenüber hätte Schenk mit dem Auto auch etwas von seinem - wie Klaus Theweleit sagen würde $>$ Körperpanzer< (vgl. Theweleit 2000) abgegeben. Dass Schenk einfach in einen solchen amerikanischen Schlitten hineingehört, zeigt dann wenig später besonders deutlich eine Szene, in der er sich notgedrungen den Mini von Mitarbeiterin Franziska ausleiht (01:17:56-01:18:05), was zu komödienartigen Effekten der vollständigen Innenraumausfüllung des kleinen Wagens führt (01:20:46-01:21:04). Ergebnis dieses Permutationstests: Schenk ist in seinen Straßenkreuzern schon richtig aufgehoben, genauso wie Kollege Ballauf im biederen blaugrauen VW-Passat Kombi.

Der ist immerhin neueren Baujahrs, was man für das Vehikel gleichen Typs des Kieler Ermittlers Klaus Borowski nicht sagen kann. Dessen Dienstwagen, liebevoll »mein Brauner « ${ }^{6}$ genannt, ist ein seit längerer Zeit nicht mehr gebauter VW PassatKombi, der den etwas verschrobenen und durch seine Kollegin vom psychologischen Dienst unter ständiger latenter Beobachtung stehenden Kommissar als Charakterpendant jahrelang begleitete. Doch wie den Wagen wechseln, ohne damit zugleich auch das Charakterduo von Auto und Kommissar insgesamt infrage zu stellen? Die Lösung zeigt die Folge Borowski und der stille Gast (2012), in der gleich zu Beginn der Innenspiegel des geliebten Wagens abfällt (00:04:13-00:04:45). Nach rund einem Drittel der Handlung will dieser dann zunächst gar nicht mehr anspringen, fährt aber schließlich doch noch einmal, ehe er kurz vor Ende der Folge endgültig stehen bleibt, ja regelrecht zum Erliegen kommt. Kommissar und Zuschauer wissen sofort, dass hier nichts mehr zu machen ist. Folgerichtig erhält Borowskis Wagen in intertextueller Anspielung auf eine Konvention des Western-Genres von seinem Fahrer wie ein treues Pferd den Gnadenschuss (01:01:07-01:02:00). Ist das Charakterdouble von Ermittler und Vehikel damit geschieden? Keineswegs, denn gleichsam postum löst das Auto indirekt den Fall, da Borowski mit dem inzwischen in seinem Schreibtisch liegenden Innenspiegel eine seitenverkehrt geschriebene Adresse entziffern kann, die ihn zum Täter führt.

Es bleibt aber die Frage, ob mit dem >Tod< von Borowskis Wagen auch der Charakter des Kommissars gefährdet ist. Er ist es nicht, denn sein temporärer Er- 
satzwagen ist ein mausgrauer Ford (01:21:25-01:22:27), der zumindest keine neuen Charaktermerkmale für seinen Besitzer evoziert. In den danach gesendeten Folgen nutzt Borowski dann einen älteren roten Volvo (so in Borowski und der brennende Mann, 2013), von dem man annehmen darf, dass er dem Charakterbild seines Fahrers peu à peu mehr entsprechen, dessen filmische Fortsetzungsgeschichte schreiben und damit zu einem Element der Serialität werden wird. Der Dreischritt von >mein Brauner zum indifferenten mausgrauen Ford und dann zum roten Volvo, der allmählich wieder zum mit Borowski korrespondierenden Charakter aufgebaut wird, erinnert strukturell an die drei Stadien des liminalen Initiationsprozesses, wie Victor Turner sie beschrieben hat, nämlich erstens Krisenphase der alten Identität mit allmählicher Ablösung von bestehenden Strukturen (>mein Brauner tens Zwischenphase der Liminalität oder Transformation, in der bisherige Konventionen und Verhaltensmuster aufgelöst und neue ausprobiert werden (>mausgrauer Ford <); drittens Phase der postliminalen Festigung einer neuen Communitas (>roter Volvo<) (vgl. Turner 2005: 94-158).

Vor dem Hintergrund dieser vielfachen Korrelation von Kommissaren und Vehikeln erklärt sich dann auch schnell, warum die Fahrer nicht so einfach wechseln können, warum immer ein bestimmter Ermittler fährt, sehr viel seltener aber sein Partner oder seine Partnerin. Ausnahmen bestätigen dabei die Regel, da sie nur ausgesprochen selten vorkommen. Denn wenn die Autos und die Charaktere so stark aneinander gekoppelt sind, dann wäre ein Austausch zwischen beiden für die Serie ziemlich fatal. Nur Mario Kopper gehört eben in einen 1970er-Jahre-Fiat, nur Freddy Schenk in die diversen amerikanischen Youngtimer. Diese Auto/CharakterKombinationen tendieren zudem in manchen Tatorten zur paradigmatischen Expansion. So kommen im Falle des Münsteraner Pathologen Boerne, der de facto die Rolle eines Co-Ermittlers einnimmt, zum stets exquisiten Oberklasse-Fahrzeug auch noch Opernmusik (bevorzugt Wagner) und teurer Rotwein hinzu.

Haben die gefahrenen Wagen - wie im Falle von Lena Odenthal - einmal keinen >Charaktermehrwert<, dann können immerhin die Nummernschilder semantisiert werden. In der Folge Die Neue (1989) lautet es bspw. »LU-PA 618« (01:19:48), wenn man so will also >Wölfin<, wobei das »PA« im Kontext der Handlung auch als >Penthesilea<-Anspielung gelesen werden kann. ${ }^{7}$ Und wie sieht es bei den anderen Ermittlerteams aus? Die Frankfurter Kommissarin Conny Mey fährt passend zum stets etwas schrägen Outfit und insbesondere zu den roten Lackstiefeln - einen roten Golf GTI; Ulrich Tukur als verschroben-exotischer Kommissar Murot seit der ersten Folge Wie einst Lilly (2010) einen nicht minder exotischen, seit 1977 nicht mehr gebauten NSU Ro 80 mit Wankelmotor. ${ }^{8}$ Der Duisburger KultErmittler Horst Schimanski fuhr demgegenüber meist gar nicht, sondern ließ den

7 Ich danke Stefan Scherer für diesen Hinweis. 
verkappten Ästheten und Spießbürger Tanner chauffieren. Sah man Schimanski aber doch einmal am Steuer, dann war es in den ersten Folgen das eines wuchtigen Ford Granada, später das eines Citroen CX. ${ }^{9}$

Dann gibt es da noch die Wiener Kommissarin Bibi Fellner, die schon mal mit dem 94iger Pontiac ihres Freundes >Inkasso Heinzi< aus dem Zuhältermilieu aufkreuzt. Wie systematisch, ja geradezu schulbuchmäßig das Auto dabei als Mittel zur Charakterisierung der Figur eingesetzt wird, zeigt die Folge Vergeltung (2011), in der Fellner als neue, alkoholabhängige, vorher bei der >Sitte< tätige Assistentin von Kommissar Moritz Eisner eingeführt wird. Als auch für die Zuschauer ganz neue Figur kommt sie gleich zu Beginn dieser Folge mit dem getunten und auffällig lackierten amerikanischen Sportwagen im Zuhälterstil ins Bild. In engstem zeitlichem Rahmen leistet das mehr als längliche Charaktererläuterungen, aufwändige Rückblenden oder der zeitraubende Aufbau eines Charakters durch typische Handlungen. Über die gesamte Folge hinweg wird dieses Auto und mit ihm auch hier wieder das Charakterbild der Ermittlerin dann immer wieder einmal thematisiert, und zwar so, als sollte die Charakterkombination Auto/Ermittlerin mit den $\mathrm{Zu}$ schauern regelrecht eingeübt werden (Vergeltung, 00:09:07-00:10:08, 00:13:2600:14:28, 00:17:56-00:18:06, 00:26:23-00:27:35, 00:32:15-00:33:12, 01:08:2501:08:55). Im Gegensatz zu seiner Assistentin fährt Kommissar Moritz Eisner selbst einen vergleichsweise unscheinbaren weißen Golf III, den er bei einem dramaturgisch notwendigen Unfall zu Schrott fährt, ${ }^{10}$ was auch hier die Frage des Ersatzes aufwirft: Wieder ein Golf, aber diesmal ein dunkelblauer GTI. Die Charakterkorrespondenz Kommissar/Auto bleibt bestehen und mit ihr die Interesse und Spannung erzeugende Distanz zwischen den Charakteren von Kommissar und Assistentin, die ebenso gestärkt wird wie diejenige zwischen Golf und >Milieu<Vehikel. Egal ob einer der beiden Wagen oder eine der beiden Ermittlerfiguren in

8 Vgl. Claudia Arthen. »Murots Oldtimer: Das Beste an Tukurs >Tatort «.« news.de, 5. Dezember 2011 (http://www.news.de/medien/855248765/dienstwagen-affaeren-dieautos-der-tatort-kommissare/1/); [Anon.] »Mey, Murot \& Co. Die Autos der >Tatort<Ermittler.« ksta.de, 11. Juni 2013 (http:www. ksta.de/ratgeber/mey--murot---co--dieautos-der-tatort--ermittler, 15189524,12027466.html).

9 Florian Gerber. »Reihe: >Tatort<-Kommissare und ihre Autos. Heute: Horst Schimanski alias Götz George.« auto.de, 17. Januar 2012 (http://www. auto.de/blog/showblog/ entryId/1978/Reihe-Tatort-Kommissare-und-ihre-Autos-Heute-Horst-Schimanski-aliasGoetz-George).

10 Gegenstand der Folge sind Morde an als gewalttätig aufgefallenen Jugendlichen in der UBahn, an Bushaltestellen etc. Damit der Kommissar dieses Umfeld selbst kennenlernt, muss er gleich zu Beginn einen Unfall haben und zum Busfahrer werden. 
den kommenden Folgen auftaucht: Immer wird die gesamte Viererstruktur mit thematisiert.

Bleibt nach Österreich noch der Blick in die Schweiz. Die am Bodensee spielenden Konstanzer Tatort-Folgen haben vor einiger Zeit ein Schweizer Pendant mit dem Handlungsort Luzern bekommen, wohin der vormals am Bodensee tätige Schweizer Kommissar Reto Flückiger versetzt wurde. In der zweiten Folge dieses neuen Tatort-Tatortes mit dem Titel Skalpell (2012) trat seine zuvor in Los Angeles tätige Assistentin Liz Ritschard mit für die Schweiz neuen amerikanischen Ermittlungsmethoden auf, brachte sie doch ein Notebook mit an den Tatort (und der war immerhin im Wald). Stück für Stück wurde ihr Charakterbild in dieser Folge als >amerikanisch< aufgebaut, und zwar mit - wie könnte es anders sein - dem Kulminationspunkt der fast schon an ein Geständnis erinnernden Offenlegung des von ihr gefahrenen Autos. Denn als Flückiger mit seiner Assistentin verabredet, dass sie ihn abholt, kurvt sie mit einem geradezu riesig anmutenden Chevrolet Coupé durch die engen Gassen der Luzerner Altstadt. Größer könnte der Charaktergegensatz von modern-amerikanisch ermittelnder Assistentin und traditionell-schweizerisch vorgehendem Kommissar (der auch hier wieder einen älteren Passat-Kombi nutzt) kaum inszeniert werden. Und wenn dann sowohl Kommissar als auch Assistentin jeweils kulturpsychologisch philosophieren: »Zeige mir Dein Auto und ich sage Dir, wer Du bist « (Skalpell, 00:20:55-00:21:15 und 00:49:00-00:50:03), dann hat auch der letzte Zuschauer verstanden, dass Kommissare und ihre Autos eine semantische Einheit bilden. ${ }^{11}$

Auf einer subdominanten Ebene, die noch eine eigene Untersuchung wert wäre, kommt bisweilen noch der Fahrstil hinzu, der immer wieder Anlass bietet, die Charaktere innerhalb der Ermittlerteams gegeneinander abzugrenzen. Und ganz gelegentlich gibt es auch so etwas wie Auto-Zweikämpfe, die in der Regel ebenfalls an die antagonistischen Charaktere der Fahrer bzw. Ermittler zurückgebunden sind. ${ }^{12}$ All das ließe sich auch noch auf der Ebene der Nebenfiguren fortsetzen. Zugespitzt formuliert: Der böse reiche schwule Architekt und Mörder fährt einen nagelneu glänzenden schwarzen Bentley, und der aus völliger sozialer Verzweiflung heraus

11 Die ist im Falle von Liz Ritschard auch dadurch nicht mehr aufzuheben, dass sie am Ende des Dialogs darauf verweist, das Auto gehöre ihrem Bruder.

12 Wie in einem Showdown stehen sich in Letzte Tage (2013) die Autos der deutschen Kommissarin und des Schweizer Ermittlers gegenüber; ihr silberfarbener MercedesKombi und sein sandfarbener Geländewagen (Letzte Tage, 00:13:00-00:15:00); in Tödliche Tarnung (2009) gibt es eine Zweikampf-Situation an einer Ampel, die Kommissar Thorsten Lannert dadurch löst, dass er das Blaulicht auf das Dach seines rostbraunen Porsches stellt und betont langsam anfährt (00:11:45-00:12:15) (für diesen Hinweis danke ich Christian Hißnauer). 
einen kleinen Lottoladen überfallende Familienvater einen notdürftig zusammengeflickten Opel Kadett undefinierbarer Farbe.

Schließlich gibt es auch Übergänge von den human-automobilen Charakteren zur Handlungsebene, und zwar in Form von Korrespondenzen zwischen gescheiterten Autos und gescheiterten Menschen. Es sind immer die gescheiterten Existenzen, die mit eigentlich schon längst im Verkehr gescheiterten alten Autos dieses Scheitern noch einmal performativ durchspielen. ${ }^{13}$ Der nicht-normale Lebenslauf der Opfer wird so zur symbolisch lesbaren nicht-normalen Autofahrt, zum automobilen Lebensweg. ${ }^{14}$ Das wird bisweilen als regelrechter Kreislauf inszeniert, was der Köln-Leipziger Doppel-Tatort Kinderland/Ihr Kinderlein kommet (2012) fast schon zu plakativ aufzeigt: Mit vom Schrottplatz gestohlenen Autos werden Mädchen entführt, umgebracht, dann wie Müll im Rhein entsorgt oder mit den zugleich als Tatwerkzeugen wie auch als Tatorten fungierenden Autos wieder auf den Schrottplatz zurückgebracht.

Was bedeutet all das nun für die Frage der Serialität? Die Autos in Kombination mit den sie jeweils fahrenden Ermittlern bilden im Tatort eine Art gesamtgesellschaftliche Matrix von intuitiv nachvollziehbaren Charakter- und Sozialpositionen, die bereits im medialen Alltag regelrecht eingeübt werden und die im Tatort nur noch abgerufen bzw. aktualisiert werden müssen. Dieses System der Charakteranalogien zwischen Autos und Ermittlern beruht auf relativ strikt durchgehaltenen Distinktionen. Eine einmal besetzte Kombination kann nicht an einem anderen Ort ein zweites Mal genutzt werden (was im öffentlich-rechtlichen Fernsehen tendenziell sogar serienübergreifend gilt). ${ }^{15}$ Auf diese Weise ist mit einer bereits realisierten Position im Hintergrund aber stets das ganze System der schon vergebenen Positionen als Wissensfundus präsent, was aus Rezipienten-Perspektive einen Zusammen-

13 In der NDR-Folge Kurzschluß (1975) bleibt das Fluchtauto eines Bankräubers wegen einer defekten Benzinleitung stehen (00:07:00-00:07:28). Daraufhin wechselt er in den verbeulten Ford Taunus Kombi eines abgetakelten Vertreters und zwingt diesen, ihn zu fahren.

14 Vgl. zum Narrativ der >nicht-normalen Fahrt< die Beiträge in Gerhard/Grünzweig/ Link/Parr (2003). - Auf einem Schrottplatz (der Geschichte?) beginnt auch die erste Folge des MDR-Ermittlerteams Ehrlicher/Kain (Ein Fall für Ehrlicher, 1992, 00:00:0100:02:10).

15 Über die Vielzahl der Ermittlerteams der letzten vierzig Jahre hinweg ist es immer schwieriger geworden, noch originelle Kombinationen von Ermittlern und Autos zu finden. Daher wird - wie im Falle von Tatort-Münster und Tatort-Saarbrücken - das Spektrum der Vehikel erweitert: Thiele ermittelt in Münster mit dem Fahrrad, dem Taxi seines Vaters und den Edelkarossen des Pathologen Boerne; Stellbrink in Saarbrücken mittels Motorroller und Auto. 
hang über die einzelnen Folgen und die einzelnen regionalen Tatort-Serien hinweg auch für den Tatort als Reihe aus Serien insgesamt stiftet, und zwar sowohl im synchronen Schnitt durch die je aktuellen Tatort-Serien als auch in der diachronen Perspektive ihrer 40-jährigen Geschichte.

Jedes Thematisieren von Autos (oder der Ersatzvehikel) im Tatort ist damit immer auch eine Form der Reflexion des Seriencharakters. Denn als Formen »variierende[r] Wiederholung « als Basis von Serialiät (vgl. Kelleter 2012: 12; Krah 2010: 87; Cuntz 2012: 242), sind Autos Teil desjenigen >Kitts<, der Tatort auf beiden Ebenen zusammenhält. Krah (2010: 87f.) hat darauf hingewiesen, dass serielles Erzählen v.a. die Frage nach der/den Textgrenze/n bzw. deren Überschreitung aufwirft. Das >Auto< wäre dann ein Element, das genutzt wird, um Sub-Narrationen in Form von fortlaufend lesbaren short cuts $^{16} \mathrm{zu}$ realisieren, die mehrere Folgen übergreifen und im Tatort auf der Ebene der je regionalen Serien angesiedelt sind. Gleichzeitig wird auf einer nicht-narrativen Ebene aber durch den die regionalen Serien übergreifenden Charakter der >Auto-Matrix < auch die Grenze in Richtung Gesamtreihe Tatort und sogar darüber hinaus überschritten. Das ist u.a. möglich, weil die Matrix der Auto-Charaktere mit Blick auf die Gesamtgesellschaft als eine Art Pars-pro-toto-Modell der Gesellschaft in der Ausdifferenzierung ihrer nicht immer nur feinen Unterschiede (vgl. Bourdieu 1988) fungiert. Damit aber sind Autos nicht unwichtige Elemente auch der Personalisierung von Werten und derjenigen Ideologien, in die sie eingebunden sind. ${ }^{17}$

\section{SPRITZTOUR IN DIE REGIONALITÄT}

Eine weitere, ganz andere Funktion von Autos im Tatort ist diejenige der regionalen räumlichen Verankerung. Sie erfolgt erstens dadurch, dass mit Vorliebe dann Nummernschilder von Autos eingeblendet werden, wenn gezeigt werden soll, wo in einer Region man sich gerade befindet. Dieses Verfahren wurde stärker in den 1970er und 1980er Jahren genutzt als in den 2000er Jahren. In jüngster Zeit wird darauf - nachdem es für einige Zeit eher vermieden wurde - aber wieder stärker zurückgegriffen. Derselbe Effekt regionaler Lokalisierung wird durch das signifikante In-Szene-Setzen regional verorteter Automarken erzielt: Im Münchener Tatort wird BMW gefahren, in Stuttgart nutzt der Kommissar einen 1974er Porsche 911 Targa, der in fast jeder Folge wenigstens einmal in Großaufnahme vor Stuttgarter Kulisse

16 Vgl. zu short cuts und seriellem Erzählen den Beitrag von Moritz Baßler in diesem Band sowie Krah 2010: 93 und Nies 2007.

17 An Autos festgemachte soziale Unterschiede werden z.B. in Feuerteufel (2013) thematisiert. 
im Hintergrund zu sehen ist. Diese Form der Regionalisierung ${ }^{18}$ hat dem Tatort gelegentlich den Vorwurf des Produkt-Placements eingebracht, ${ }^{19}$ was zur Folge hatte, dass die Marken - etwa im Bremer Tatort - ausgetauscht wurden: Mercedes gegen Audi. $^{20}$

Weiterhin kann die Funktion der regionalen Verankerung dadurch erfüllt werden, dass bei Autofahrten durch Städte nicht die direkten Wege genommen werden, sondern solche, auf denen die touristischen >most famous view points< abgearbeitet werden, um dann von den Zuschauern vor dem Bildschirm wiedererkannt werden zu können. Das lässt sich besonders gut an den Kölner, etwas weniger an den Münchner und besonders deutlich an den Berliner Tatort-Serien beobachten: Egal wohin es geht, wiedererkennbare >landmarks` wie die Feldherrnhalle, der Kölner Dom oder der Alexanderplatz werden wenigstens kurz gezeigt, ${ }^{21}$ und zwar auch dann, wenn es im Falle von Berlin gilt, von - zugespitzt formuliert - Zehlendorf nach Dahlem zu fahren. Das lässt sich generalisieren: »Das ARD-Format Tatort bildet mit der Realität unterschiedlicher Räume die Eigenlogik deutscher Städte ab, die es in der Abbildung selbst erst erzeugt." (Scherer/Stockinger 2010: 31)

Regionalisierungseffekte durch Autos können aber auch sehr viel indirekter evoziert werden. So kann man die Tatsache, dass Schenk als Polizeibeamter im Kölner Tatort mit Vorliebe amerikanische Wagen fährt, die zuvor beschlagnahmt wurden, unter regionalem Aspekt auch als eine recht gelungene Illustration dessen ansehen, was der Volksmund >Kölner Klüngel< zu nennen pflegt, nämlich die zwar

18 Prinz und Wiendl 2010 haben gezeigt, dass Tatorte umso erfolgreicher sind, je besser die Verankerung in der Regionalität gelingt.

19 Vgl. dazu Joachim Schmitz. »Die Kommissarin und das Auto - Produktplatzierung im Tatort?« noz.de, 19. Dezember 2011 (http://www.noz.de/deutschland-undwelt/kultur/fern sehen/59570025/die-kommissarin-und-das-auto-produktplatzierung-im-tatort); Kathrin Buchner. »Kritik an der ARD. Schleichwerbung beim >Tatort $\ll$ ? stern.de, 26. Februar 2009 (http://www.stern.de/kultur/tv/kritik-an-der-ard-schleichwerbung-beim-tatort-655 979.html).

20 Für die Auswahl der Autos verantwortlich sind - so Melanie Wolber vom SWR BadenBaden im Rahmen ihres Konferenz-Vortrags - die jeweiligen Redakteurinnen und Redakteure, wobei zum einen nach dem Charakterbild der Ermittler selektiert, zum anderen dem Prinzip der Markenstreuung gefolgt wird. Um dem Vorwurf des Produkt-Placements vorzubeugen, benutzte der SWR zudem seit einiger Zeit nur Automodelle, die aktuell nicht mehr im Handel sind. - Andere Sender wechseln inzwischen vermehrt die Fahrzeuge und Marken.

21 Vgl. zu den »Geographien des Fernsehens« mit Blick auf die Kölner Tatorte Böllhofer 2007; siehe generell Griem/Scholz 2010 sowie zum Münchner Tatort Scherer/Stockinger 2010. 
offiziell nicht vorgesehenen, aber dennoch allerorten praktizierten Verbindungen zwischen Öffentlichkeit und Privatheit, formellen und informellen Institutionen und Instanzen, die eigentlich nicht zusammenarbeiten dürfen, es unter der Hand dann aber doch tun. Über das Auto wird der >kölnische< Charakter somit in der Region verankert.

\section{Automobile Raumdramaturgien}

$\mathrm{Zu}$ den räumlich-dramaturgischen Funktionen von Autos im Fernsehkrimi gehört zunächst einmal, dass Autos als mobile Tatorte dienen, die zwischen verschiedenen Räumen hin und her bewegt werden können. Hier haben wir es zugleich mit der Einheit und Verschiedenheit von Orten im Sinn der aristotelischen Dramaturgie zu tun. Weiter dienen Autos der Trennung bzw. Unterscheidung einer Innen- von einer Außenwelt (etwa im Falle der immer wieder vorkommenden mobilen Abhörstationen in Kleintransportern). Auch hier sind es wieder zwei Orte, derjenige des verlängerten Polizeibüros und derjenige der jeweils beobachteten Wohnung bzw. des jeweils beobachteten Tatorts, die im Inneren des Kleintransporters durch die Abhörtechnik, die heute stets auch eine Abseh-Technik ist, zusammengeführt werden können: wiederum die gleiche Dramaturgie der Einheit und zudem auch noch gleichzeitigen Präsenz zweier verschiedener Orte, rückgebunden an das Vehikel Auto (vgl. stellvertretend Spuk aus der Eiszeit, 1988, 01:07:50-01:13:26; Herzversagen, 2004, 01:19:40-01:22:06).

Eine andere dramaturgische Funktion von Autos im Tatort ist es, das Büro mit dem Tatort zu verkoppeln. Die Polizeikommissare des Fernsehens scheinen nämlich entweder im Büro zu sitzen und dort zu telefonieren, oder im Auto, um dort ebenfalls zu telefonieren. Das Auto wird so zum verlängerten Büro, in dem begonnene Dialoge fortgeführt werden können. Wenn das Auto aber auch Büro ist, dann ermöglicht es in dramaturgischer Hinsicht hier ebenfalls wieder so etwas wie Bewegung im Raum bei gleichzeitiger Wahrung der Einheit des Ortes und der Handlung. Auf diese Weise gelingt dem Fernsehkrimi etwas, um das ihn eigentlich mancher (traditionelle) Theatermacher beneiden müsste. Und schließlich ist der Wagen noch der Ort, an dem sich niemand der Kommunikation so einfach entziehen kann. Symptomatisch sind dafür die sehr langen >Im-Auto-Dialoge< in den Frankfurter Tatorten mit dem Ermittlerteam Dellwo/Sänger (bspw. in Oskar, 2002, 01:11:5001:13:00).

Schließlich werden mit Hilfe von Autofahrten Landschaften und Räume nicht nur in Szene gesetzt, sondern auch als semantisch distinkte Räume im Sinne Jurij M. Lotmans (1972: 311-329) etabliert. Das ist gleich 1970 in Taxi nach Leipzig der Fall, wenn der westdeutsche Kommissar aus seinem Ford 17M in einen Wartburg 
der Volkspolizei wechselt und erst dann den Sonderraum der Transitstraße verlässt und ins ländliche Niemandsland der DDR fährt (00:44:00-00:46:00). Regelmäßig trifft man diese Form der semantischen Konstitution von Räumen durch Autofahrten in den Hannoveraner Tatorten an, die weitläufigere ländliche Schauplätze durch Autofahrten als Bühne des Geschehens etablieren, wodurch »die Realität unterschiedlicher Räume« nicht lediglich abgebildet wird, sondern diese Räume überhaupt erst hervorgebracht werden (Scherer/Stockinger 2010: Abschn. 4). D.h., Räume sind »nicht nur Bühne«, sondern auch »kollektive[] Akteur[e]« (Griem/ Scholz 2010: 20), aktive Mitproduzenten. Bei den Hannoveraner Tatorten wird das dadurch deutlich, dass sie vielfach zwischen den beiden Raumkonzepten, die Scherer und Stockinger als »Realismus des Ländlichen« und als »Realismus des [...] Mythischen « (2010: 4) unterschieden haben, hin und her wechseln, ja nicht selten sogar das eine gegen das andere ausspielen, etwa indem sich entgegen der im Alltag üblichen Semantisierungen gerade die ländliche Idylle als Raum der Kriminalität entpuppt. An der Konstitution beider haben Autos und Autofahrten keinen geringen Anteil, denn sie übernehmen es häufig, die Perspektive für den Establishing Shot von Landschaften und Räumen herzustellen.

\section{AUSROLLEN LASSEN: WAS Autos IM TATORT NOCH LEISTEN}

Einige Auto-Funktionen sollen abschließend nur noch kurz aufgezählt werden. Da$\mathrm{zu}$ gehört erstens die therapeutische Verwendung des Autofahrens in Form von normalisierenden Autofahrten mit Mensch/Maschine-Symbiosen, wie man sie in der Literatur seit Erich Maria Remarques Roman Drei Kameraden kennt (Remarque 1938; dazu Parr 1998). Dabei gewinnt eine Figur mit der Kontrolle über das technische Vehikel auch die über die eigene Psyche zurück. Typischer Dialog im TV-Krimi: >Und dann sind sie einfach so durch die Gegend gefahren?<>Ja, um mich zu beruhigen. $<>$ Hörst du, er ist einfach so durch die Gegend gefahren. $<>$ Ja, um sich zu beruhigen. $<$ Zweitens sind Autos besonders geeignete Orte, um Antagonismen auf engstem und zudem geschlossenem Raum zusammen zu bringen, etwa Entführer und Entführte, Verdächtige und Kommissar, Kommissar und Opfer.

Drittens entspricht dem Stopp des Autos in der Dramaturgie oft das, was Heinrich Böll noch als Ende einer Dienstfahrt bezeichnet hätte (Böll 1966): Kommissare, die frustriert von ihrem Job oder anderweitig psychisch angeschlagen sind, steigen häufig symbolisch aus einem Auto aus. Autos sind viertens Orte der Verknüpfung des Sichtbaren mit dem eigentlich Unsichtbaren (Entführter im Kofferraum, Entführer am Steuer). Fünftens leisten sie häufig Kopplungen von >Dynamik< und 
>Stillstand<, d.h. auf dem Weg über Autos kann hochgradig plausibel zwischen diesen beiden Zuständen gewechselt werden und zwar durchaus auch ad hoc.

Sechstens und letztens: Autos sind häufig die dramaturgischen Schnittpunkte zwischen der eigentlichen Krimihandlung, der Dienstgeschichte und den sporadisch in kurzen Einschüben erzählten Privatgeschichten der Ermittler, sofern diese nicht ohnehin als Parallelgeschichten angelegt sind, die die Ermittler auch mit ihrer privaten Seite in den Fall involvieren (vgl. als Beispiel Letzte Tage, 2013, 00:00:3000:01:30). Da die Privatgeschichten eher folgenübergreifend realisiert werden, also fortlaufende Handlungsstränge darstellen (vgl. Hißnauer/Scherer/Stockinger 2012: 147), sind es auch hier wieder die Autos, die zugleich die konzise Verankerung im Geschehen der einzelnen Folge garantieren, über mehrere Folgen hinweg aber auch Serialität im Sinne des von Lorenz Engell beschriebenen >operativen Gedächtnisses< einer Serie. Denn die Serie ist im Fernsehen

»dasjenige Format, in dem das Fernsehen seine Funktion als operatives Gedächtnis erstens ausübt und implementiert, zweitens erprobt, variiert und, in einem durchaus evolutionären Sinne, fortentwickelt und drittens reflektiert. Für das Fernsehen ist die Serie mithin schließlich das funktionale Äquivalent zu einer Theorie des Gedächtnisses« (Engell 2010: 116).

\section{ABBREMSEN, EINPARKEN}

Auch wenn Autos im Tatort vielfältige Funktionen übernehmen, wird man doch einschränkend sagen müssen, dass Automobile genutzt werden können, aber nicht genutzt werden müssen, denn die aufgezeigten Funktionen können auch in anderer Form und mit anderen Mitteln erzielt werden. Weiter wäre über die lange Laufzeit der Reihe Tatort hinweg inzwischen auch historisch zu differenzieren: hinsichtlich der ästhetischen Entwicklungen und Vorlieben, hinsichtlich der technischen Möglichkeiten und im Hinblick auf den Status von Serien im öffentlich-rechtlichen Fernsehen usw. So zeigen viele der älteren Folgen aus den frühen 1970er Jahren durchaus lange Autofahrten in der Landschaft. In den mittleren und späten 1970er und noch in den 1980er Jahren gibt es dann immer wieder eine Tendenz zum Kammerspiel (vgl. Leder 1997: 8), v.a. bei den Haferkamp-Tatorten aus Essen oder den Veigl-Tatorten aus München, in denen in der Regel hauptsächlich im und vom Polizeibüro aus agiert wird. Autos werden damit zu eher randständigen Gegenständen. Das ändert sich mit Schimanski, einem Kommissar für >draußen<, der - wenn nicht gerade betrunken - ständig in irgendeiner Form von Bewegung ist und »eine neue Bewegungsfreiheit« geradezu »vorzuführen« scheint (Koebner 1990: 22) - nicht zuletzt im und mit dem Auto. Der Wechsel vom »Kammerspiel « zum »motion picture $«$, wie ihn Leder (1997: 10) anhand des Übergangs von Haferkamp zu Schi- 
manski für den Ruhrgebiets-Tatort konstatiert, hat damit auch zur Vervielfältigung derjenigen Funktionen geführt, die Autos im Tatort übernehmen können. Aber diese historische Entwicklungslinie aufzuzeigen, würde einen eigenen Beitrag nötig machen.

\section{LITERATUR}

Bierbaum, Stefanie. >Tatort Telefon<. Mit dem Anruf kommt der Tod. Das Telefon im Kriminalfilm: Eine Analyse für den >Tatort $<$. Bauhaus Universität Weimar, Fakultät Medien: Forschungsarbeit im Wintersemester 2000/2001.

Böll, Heinrich. Ende einer Dienstfahrt. Erzählung. Köln, Berlin: Kiepenheuer \& Witsch, 1966.

Bollhöfer, Björn. Geographien des Fernsehens: Der Kölner >Tatort< als mediale Verortung kultureller Praktiken. Bielefeld: transcript, 2007.

Bourdieu, Pierre. Die feinen Unterschiede. Kritik der gesellschaftlichen Urteilskraft. 2. Auflage. Frankfurt a.M.: Suhrkamp, 1988.

Cuntz, Michael. »Seriennarrativ (TV).«Handbuch der Mediologie. Signaturen des Medialen.« Christina Bartz, Ludwig Jäger, Marcus Krause und Erika Linz (Hg.). München: Fink, 2012. 242-252.

Engell, Lorenz. »Erinnern/Vergessen. Serien als operatives Gedächtnis des Fernsehens.«Serielle Formen. Von den frühen Film-Serials zu aktuellen Quality-TVund Online-Serien. Robert Blanchet, Kristina Köhler, Tereza Smid und Julia Zutavern (Hg.). Marburg: Schüren, 2011. 115-132.

Griem, Julika und Sebastian Scholz. »Beweisaufnahme: Zur medialen Topographie des >Tatort<.«Tatort Stadt. Mediale Topographien eines Fernsehklassikers. J.G. und S.S. (Hg.). Frankfurt a.M.: Campus, 2010. 9-28.

Guillaume, Marie-Ange und François Roca. Hoppla Hugo. Hildesheim: Gerstenberg, 2003.

Hißnauer, Christian, Stefan Scherer und Claudia Stockinger. »Formen und Verfahren der Serialität in der ARD-Reihe >Tatort<.« Populäre Serialität: Narration Evolution - Distinktion. Zum seriellen Erzählen seit dem 19. Jahrhundert. Frank Kelleter (Hg.). Bielefeld: transcript, 2012. 143-167.

Horváth, Ödon von. Kasimir und Karoline. Frankfurt a.M.: Suhrkamp, 1994.

Kelleter, Frank. »Populäre Serialität. Eine Einführung.«Populäre Serialität: Narration - Evolution - Distinktion. Zum seriellen Erzählen seit dem 19. Jahrhundert. F.K. (Hg.). Bielefeld: transcript, 2012. 11-46.

Klippel, Heike und Hartmut Winkler. »>Gesund ist, was sich wiederholt $<$. Zur Rolle der Redundanz im Fernsehen."Aspekte der Fernsehanalyse. Methoden und Modelle. Knut Hickethier (Hg.). Münster: LIT, 1994. 121-135. 
Koebner, Thomas. »>Tatort< - zu Geschichte und Geist einer Kriminalfilm-Reihe.« Augen-Blick. Marburger Hefte zur Medienwissenschaft 9: >Tatort<. Die Normalität als Abenteuer (Dezember 1990): 7-31.

Krah, Hans. »Erzählen in Folge. Eine Systematisierung narrativer Forschungszusammenhänge.« Strategien der Filmanalyse - reloaded. Festschrift für Klaus Kanzog. Michael Schaudig (Hg.). München: diskurs film, 2010. 85-114.

Leder, Dieter. »Ortsbegehungen. Diskussionsreihe Krimi: Der >Tatort< des WDR.« Funk-Korrespondenz (1997), H. 40: 3-13.

Legal, Claus und Hans-Wilhelm Saure. >Derrick«. »Harry, hol schon mal den Wagen.« Berlin: Ullstein, 1998.

Link, Jürgen und Siegfried Reinecke. »>Autofahren ist wie das Leben<. Metamorphosen des Autosymbols in der deutschen Literatur.«Technik in der Literatur. Harro Segeberg (Hg.). Frankfurt a.M.: Suhrkamp, 1987. 436-482.

Lotman, Jurij M. Die Struktur literarischer Texte. München: Fink, 1972.

Nies, Martin. »Short Cuts - Great Stories: Sinnvermittlung im filmischen Erzählen in der Literatur und literarischem Erzählen im Film.«Kodikas/Code. Ars Semiotika 30 (2007), H. 1/2: 109-135.

Gerhard, Ute, Walter Grünzweig, Jürgen Link und Rolf Parr (Hg.). (Nicht) normale Fahrten. Faszinationen eines modernen Narrationstyps. Heidelberg: Synchron, 2003.

Parr, Rolf. »Tacho. km/h. Kurve. Unfall. Körper. Erich Maria Remarques journalistische und kunstliterarische Autofahrten.«Erich Maria Remarque. Leben, Werk und weltweite Wirkung. Thomas F. Schneider (Hg.). Bramsche: Universitätsverlag Rasch, 1998. 69-90.

Parr, Rolf. »Verkehr, Auto und zwei Grenzen der Normalität in den Romanen Alexander Lernet-Holenias.«Alexander Lernet-Holenia. Poesie auf dem Boulevard? Thomas Eicher und Bettina Gruber (Hg.). Köln, Wien: Böhlau, 1999. 209-223.

Parr, Rolf. »Auto/Wagen.«Metzler Lexikon literarischer Symbole. Günter Butzer und Joachim Jacob (Hg.). 2. erweiterte Auflage. Stuttgart, Weimar: Metzler, 2009. 35-36.

Prinz, Joachim und Andreas Wiendl. »Bestimmungsgründe für den Erfolg von TVKrimis: Das Beispiel >Tatort $<. « M e d i e n w i r t s c h a f t$. Zeitschrift für Medienmanagement und Kommunikationsökonomie (2010), H. 1: 20-29.

Remarque, Erich Maria. Drei Kameraden. Roman. Amsterdam: Querido, 1938.

Scherer, Stefan und Claudia Stockinger. »Tatorte. Eine Typologie zum Realismus des Raums in der ARD-Reihe >Tatort< und ihre Umsetzung am Beispiel Münchens.« IASLonline, 19. Februar 2010 (http://www.iaslonline.de/index.php? vorgang_id=3166).

Theweleit, Klaus. Männerphantasien. Bd. 1 und 2. München: Piper, 2000.

Turner, Victor. Das Ritual. Struktur und Anti-Struktur. Frankfurt a.M., New York: Campus, 2005. 


\section{BEHANDELTE TATORT-FOLGEN}

Taxi nach Leipzig, 29. November 1970 (NDR, R: Peter Schulze-Rohr)

Kurzschluss, 7. Dezember 1975 (NDR, R: Wolfgang Petersen)

Spuk aus der Eiszeit, 10. Juli 1988 (NDR, R: Stanislav Barabas)

Die Neue, 29. Oktober 1989 (SWF, R: Peter Schulze-Rohr)

Ein Fall für Ehrlicher, 19. Januar 1992 (MDR, R: Hans-Werner Honert)

Bildersturm, 21. Juni 1998 (WDR, R: Nikolaus Stein von Kamienski)

Oskar, 21. April 2002 (HR, R: Nikolaus Stein von Kamienski)

Herzversagen, 17. Oktober 2004 (HR, R: Thomas Freundner)

Tödliche Tarnung, 1. März 2009 (SWR, R: Rainer Matsutani)

Vergeltung, 6. März 2011 (ORF, R: Wolfgang Murnberger)

Borowski und der coole Hund, 6. November 2011 (NDR, R: Christian Alvart)

Das Dorf, 4. Dezember 2011 (HR, R: Justus von Dohnányi)

Kinderland, 8. April 2012 (MDR/WDR, R: Thomas Jauch)

Ihr Kinderlein kommet, 9. April 2012 (WDR/MDR, R: Thomas Jauch)

Skalpell, 28. Mai 2012 (SRF, R: Tobias Ineichen)

Borowski und der stille Gast, 9. September 2012 (NDR, R: Christian Alvart)

Feuerteufel, 28. April 2013 (NDR, R: Özgür Yildirim)

Borowski und der brennende Mann, 12. Mai 2013 (NDR, R: Lars Kraume)

Letzte Tage, 23. Juni 2013 (SWR/SRF, R: Elmar Fischer)

\section{Andere Zitierte Filme, Mehrteiler, Serien Und REIHEN}

Derrick (BRD/D/AUS/CH, 1974-1998 [ZDF])

Knight Rider (USA, 1982-1986 [NBC]) 


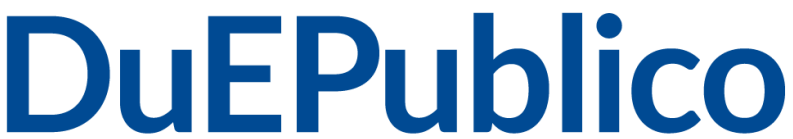

Duisburg-Essen Publications online offen im Denken

Ub \begin{tabular}{c|c} 
universitäts \\
bibliothek
\end{tabular}

Dieser Text wird über DuEPublico, dem Dokumenten- und Publikationsserver der Universität Duisburg-Essen, zur Verfügung gestellt. Die hier veröffentlichte Version der EPublikation kann von einer eventuell ebenfalls veröffentlichten Verlagsversion abweichen.

DOI: $\quad 10.14361 /$ transcript.9783839424599.129

URN: urn:nbn:de:hbz:464-20210224-172515-2

Parr, Rolf: Vehikel, Charakter-Pendant und Mittel zur Raumerkundung. Das Auto als multifunktionales Strukturelement im Tatort.

DOI: https://doi.org/10.14361/transcript.9783839424599.129

In: Zwischen Serie und Werk: Fernseh- und Gesellschaftsgeschichte im »Tatort" I

Hissnauer, Christian ; Scherer, Stefan ; Stockinger, Claudia (Hrsg.).

Bielefeld: transcript-Verlag, 2014 - PDF-ISBN 978-3-8394-2459-9.

DOI: https://doi.org/10.14361/transcript.9783839424599, S. 129-144

(C) 2014 transcript Verlag, Bielefeld. Alle Rechte vorbehalten. 